\title{
Matlab/Simulink ile Bipolar ve Unipolar PWM Kontrol Tekniklerinin Karşılaştırmalı Olarak İncelenmesi
}

\author{
Mustafa Sacid Endiz ${ }^{*}$, Ramazan Akkaya ${ }^{2}$ \\ 1* Necmettin Erbakan Üniversitesi, Mühendislik Fakültesi, Elektrik ve Elektronik Mühendisliği, Konya, Türkiye \\ (ORCID: 0000-0003-3325-5109),msendiz@erbakan.edu.tr \\ 2 Konya Teknik Üniversitesi, Mühendislik ve Doğa Bilimleri Fakültesi, Elektrik-Elektronik Mühendisliği, Konya, Türkiye \\ (ORCID: 0000-0002-6314-1500), rakkaya@ktun.edu.tr
}

(2nd International Conference on Access to Recent Advances in Engineering and Digitalization (ARACONF)-10-12 March 2021)

(DOI: 10.31590/ejosat.900868)

ATIF/REFERENCE: Endiz, M. S. \& Akkaya, R. (2021). Matlab/Simulink ile Bipolar ve Unipolar PWM Kontrol Tekniklerinin Karşılaştırmalı Olarak İncelenmesi. Avrupa Bilim ve Teknoloji Dergisi, (24), 309-313.

$\ddot{O} \mathbf{z}$

Farklı kaynaklardan elde edilen DC gücü genliği ve frekansı ayarlanabilen AC güce dönüştürebilen güç elektroniği devrelerine inverter adı verilmektedir. AC çıkış gerilimi anahtarlara uygulanan DC gerilimin uygun şekilde anahtarlanmasıyla elde edilmektedir. Anahtarların sürülmesi amacıyla kullanılan en popüler yöntem PWM tekniğidir. Bu çalışmada, inverter devrelerinde güç katındaki yarı iletken elemanların anahtarlanması amacıyla yaygın olarak kullanılan taşıyıcı tabanlı sinüzoidal PWM yöntemlerinden bipolar ve unipolar PWM kontrol teknikleri üstünlükleri yönünden incelenmiştir. Her iki kontrol tekniği Matlab/Simulink ortamında taşıyıcı ve referans sinyaller kullanılarak modellenmiş ve oluşturulan tek fazlı tam köprü inverter devresi üzerinde uygulanarak benzetim çalışmaları yapılmıştır. Çıkış gerilim sinyalinin kalitesi bakımından farklı modülasyon indekslerinde gerçekleştirilen benzetim çalışmaları sonucunda, unipolar PWM tekniğinin bipolar PWM tekniğine göre daha etkili olduğu görülmüştür.

Anahtar Kelimeler: Tam köprü İnverter, Bipolar PWM, Unipolar PWM, Toplam Harmonik Bozulma, Gerilim Stresi.

\section{Comparative Study of Bipolar and Unipolar PWM Control Techniques using Matlab/Simulink}

\begin{abstract}
Power electronic circuits that can convert DC power obtained from different sources into AC power with adjustable amplitude and frequency are called inverters. The AC output voltage is obtained by properly switching the DC voltage applied to the switches. The most popular method for driving switches is the PWM technique. In this study, bipolar and unipolar PWM control techniques, which are widely used carrier-based sinusoidal PWM methods for the switching of the semiconductor elements on the power stage in inverter circuits, have been investigated in terms of their advantages. Both control techniques have been modeled using carrier and reference signals in Matlab/Simulink environment and simulated by applying them on a single phase full bridge inverter circuit. As a result of the simulation studies performed in different modulation indexes in terms of the quality of the output voltage signal, it has been found that the unipolar PWM technique is more effective than the bipolar PWM technique.
\end{abstract}

Keywords: Full Bridge Inverter, Bipolar PWM, Unipolar PWM, Total Harmonic Distortion, Voltage Stress.

\footnotetext{
*Sorumlu Yazar: msendiz@erbakan.edu.tr
} 


\section{Giriş}

İnverterler, girişindeki DC gerilimi çıkışında istenilen genlik ve frekansta $\mathrm{AC}$ gerilime çevirebilen DC-AC dönüştürücülerdir. Devre çıkışından elde edilen AC gerilim ve frekans sabit veya değişken olabilir (Engin ve Gülersoy, 2018). İnverter girişindeki DC gerilim değiştirilerek ve inverterin kazancı sabit tutularak değişken bir AC çıkış gerilimi elde edilebilir. İnverterin DC giriş gerilimi sabit olup değişken bir AC çıkış gerilimi istendiğinde, bu inverterin kazancının değiştirilmesiyle yani PWM kontrolüyle sağlanır (Endiz ve ark., 2015). Tam köprü inverter devre şeması Şekil 1.1'de görüldüğü gibi iki ayrı yarım köprü inverterin birleştirilmesiyle meydana gelmektedir. Tek fazlı tam köprü inverter devresinde DC-AC dönüşümü 4 adet anahtarlama elemanı ve diyotlar ile sağlanmaktadır (Llorente ve ark., 2002). Yarım köprü inverter topolojisinde iki seviyeli çıkış gerilimi üretilir ve bunun için girişte orta uçlu bir gerilim kaynağ 1 kullanılmalıdır. Tam köprü inverter topolojisinde ise iki ve üç seviyeli çıkış dalga formu normal bir gerilim kaynağı ile üretilebilmektedir (González ve ark., 2007).

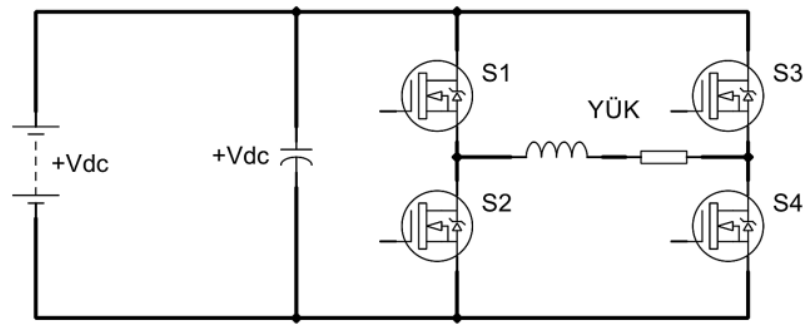

Şekil 1.1. Tam köprü inverter devre şeması

H-köprüsü olarak da bilinen tam köprü inverterde, çıkış gerilimini elde etmek için 4 farklı anahtarlama ve bir de belirsiz anahtarlama olmak üzere toplam 5 durum bulunur. Belirsiz durum, tüm anahtarların iletimde olmadığı zaman aralığında gerçekleşir. Bu durumda çıkış gerilimi $-\mathrm{V}_{\mathrm{dc}}$ ya da $+\mathrm{V}_{\mathrm{dc}}$ gerilim değerlerinden birini almaktadır (Kabalcı, 2020; Endiz ve ark., 2015).

\section{PWM Kontrol Teknikleri}

PWM tekniği, geçmişte daha çok temel elektronik devre elemanlarıyla analog olarak gerçekleştirilirken daha sonra gelişme gösteren mikrodenetleyiciler kullanılarak uygulanmaktadır. İlk yıllarda referans sinyali mikroişlemciden sağlanıp OPAMP'lı bir komparatörde taşıyıcı sinyali ile karşılaştırılarak PWM sinyali elde edilmekteydi. Sonraki yıllarda PWM sinyalleri önceden hesaplanıp bir hafiza elemanında depolanarak mikrodenetleyici yardımıyla sağlanmıştır. Günümüzde mikrodenetleyicilerin çok yüksek frekanslarda çalışır hale gelmesiyle hesaplama ve değerlendirme süreci aynı anda yapilarak PWM sinyalleri elde edilmektedir. PWM tekniğinde kare dalga darbeler oluşturulur ve oluşturulan darbelerin genişliğini değiştirmek suretiyle çıkış ana dalgasının temel bileşeni kontrol edilir. Üretilen darbelerin yarı periyottaki sayılarının arttırılması yani anahtarlama frekansının yükselmesi çıkışta istenmeyen harmoniklerin sınırlanmasını sağlar. Bunun yanında anahtarlama frekansının büyük seçilmesi anahtarlama kayıplarını arttırmaktadır (Giri ve ark., 2016; Zope ve ark., 2012). PWM sinyalleri yüksek frekanslı bir üçgen taşıyıcı dalga sinyali ile düşük frekanslı sinüzoidal referans dalga sinyalinin bir karşılaştırıcıdan geçirilmesiyle elde edilir. Taşıyıcı dalga frekansının referans dalga frekansına oranı $\mathrm{m}_{\mathrm{f}}$ ile gösterilir.

e-ISSN: 2148-2683
Üç fazlı sistemlerde $\mathrm{m}_{\mathrm{f}}$, üç ve üçün katları seçilerek hem fazlar arası denge sağlanır hem de çıkış harmonikleri sınırlanır. Sinüs sinyalinin genliğinin taşıyıcı dalga sinyalinin genliğine oranı modülasyon indeksi ile belirtilir. Modülasyon indeksinin değiştirilmesiyle çıkış geriliminin temel bileşeninin genliği değiştirilebilir. İnverterlerde kullanılan PWM teknikleri yapısı itibariyle bipolar ve unipolar olmak üzere ikiye ayrılmaktadır (Namboodiri ve Wani, 2014).

\subsection{Bipolar PWM Tekniği}

Bipolar PWM tekniğinde Şekil 2.1'de görüldüğü üzere düşük frekanslı sinüzoidal referans sinyali ve yüksek frekanslı üçgen taşıyıcı sinyal kullanılmaktadır. Referans sinyalin genliğinin taşıyıcı sinyalden büyük olduğu durumlarda çıkış lojik 1, küçük olduğu durumlarda çıkış lojik 0 olmaktadır. Bu iki sinyalin karşılaştırılmasıyla 1 ve 0 olacak şekilde elde edilen kontrol sinyalleri güç katındaki yarı iletken elemanlara uygulanmaktadır. Bu şekilde anahtarlar on-off yapılarak DC-AC güç dönüşümü sağlanmaktadır (Soomro ve ark., 2016; Khluabwannarat ve ark., 2007). Bipolar PWM tekniğinde kontrol sinyallerine göre anahtarlama durumları alttaki gibidir:

$$
\begin{aligned}
& V_{a}>V_{t} \text { ise } S_{1} \text { ve } S_{4} \text { iletimde } S_{3} \text { ve } S_{2} \text { kesimde, } V_{a c}=+V_{d c} \\
& V_{a}<V_{t} \text { ise } S_{1} \text { ve } S_{4} \text { kesimde, } S_{3} \text { ve } S_{2} \text { iletimde, } V_{a c}=-V_{d c}
\end{aligned}
$$

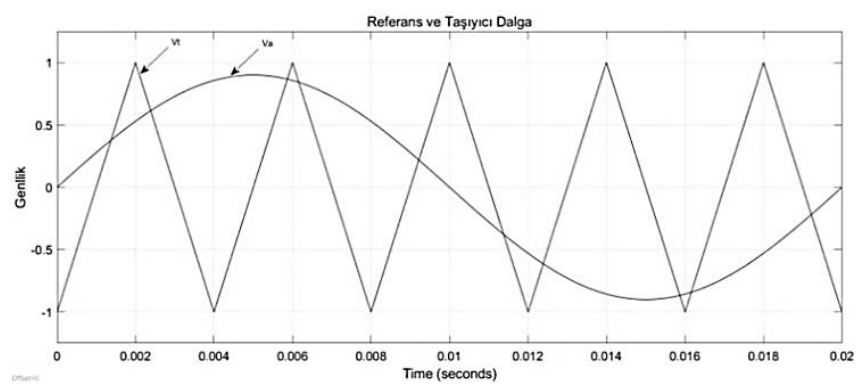

Şekil 2.1. Bipolar PWM kontrol sinyalleri

\subsection{Unipolar PWM Tekniği}

Unipolar PWM tekniğinde aralarında $180^{\circ}$ faz farkı bulunan iki adet düşük frekanslı sinüzoidal referans sinyali ve bir adet yüksek frekanslı üçgen taşıyıcı sinyal kullanılmaktadır. Bipolar PWM tekniğine benzer şekilde referans sinyallerin üçgen sinyalden büyük olduğu durumlarda çıkış lojik 1, diğer durumlarda çıkış lojik 0 olmaktadır (Wu ve ark., 2013; Sharma ve ark., 2016). Unipolar PWM tekniğinde kontrol sinyallerine göre anahtarlama durumları alttaki gibidir:

\section{$\mathrm{V}_{\mathrm{a}}>\mathrm{V}_{\mathrm{t}}$ ise $\mathrm{S}_{1}$ iletimde $\mathrm{S}_{2}$ kesimde, $\mathrm{V}_{\mathrm{ac}}=+\mathrm{V}_{\mathrm{dc}}$ \\ $\mathrm{V}_{\mathrm{a}}<\mathrm{V}_{\mathrm{t}}$ ise $\mathrm{S}_{1}$ kesimde $\mathrm{S}_{2}$ iletimde, $\mathrm{V}_{\mathrm{ac}}=-\mathrm{V}_{\mathrm{dc}}$ \\ $\mathrm{V}_{\mathrm{b}}>\mathrm{V}_{\mathrm{t}}$ ise $\mathrm{S}_{3}$ iletimde $\mathrm{S}_{4}$ kesimde, $\mathrm{V}_{\mathrm{ac}}=+\mathrm{V}_{\mathrm{dc}}$ \\ $\mathrm{V}_{\mathrm{b}}<\mathrm{V}_{\mathrm{t}}$ ise $\mathrm{S}_{3}$ kesimde $\mathrm{S}_{4}$ iletimde, $\mathrm{V}_{\mathrm{ac}}=-\mathrm{V}_{\mathrm{dc}}$}

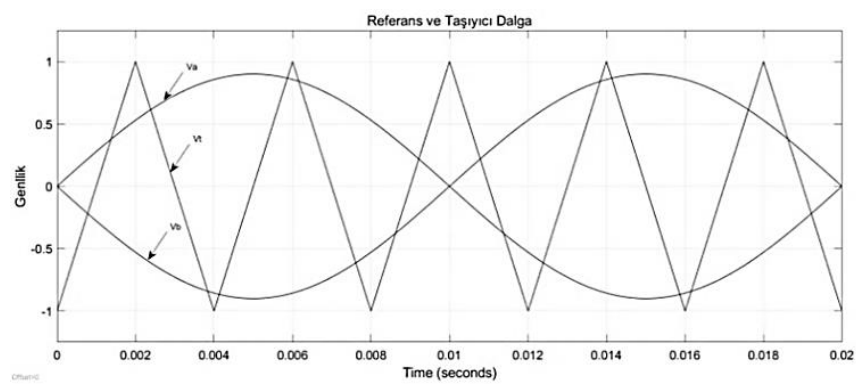

Şekil 2.2. Unipolar PWM kontrol sinyalleri 


\section{Benzetim Çalışmaları}

Bipolar ve unipolar PWM kontrol teknikleri Matlab/Simulink ortamında referans ve taşıyıcı sinyaller kullanılarak modellenmiş ve tek fazlı tam köprü inverter devresi üzerinde uygulanarak çıkış gerilim sinyalinin kalitesi bakımından benzetim çalışmaları yapılmıştır. Simulinkte referans sinyal sinüzoidal dalga üreteci, taşıyıcı sinyal testere dişi dalga üreteci kullanılarak modellenmiştir. Giriş gerilimi $310 \mathrm{~V}$, referans sinyal frekans $150 \mathrm{~Hz}$, taşıyıcı sinyal frekansı $1 \mathrm{kHz}$ ve modülasyon indeksi sirasıyla 0.85 ve 1 olarak seçilmiştir. Yapılan benzetim çalışmalarında farklı modülasyon indekslerinin seçilmesinin temel nedeni çıkış gerilim sinyalinin kalitesinin değişimini daha iyi gözlemlemektir. Şekil 3.1 ve Şekil 3.2'de sirasıyla bipolar PWM kontrol tekniği için oluşturulan Matlab/Simulink modeli ve dalga şekilleri görülmektedir.

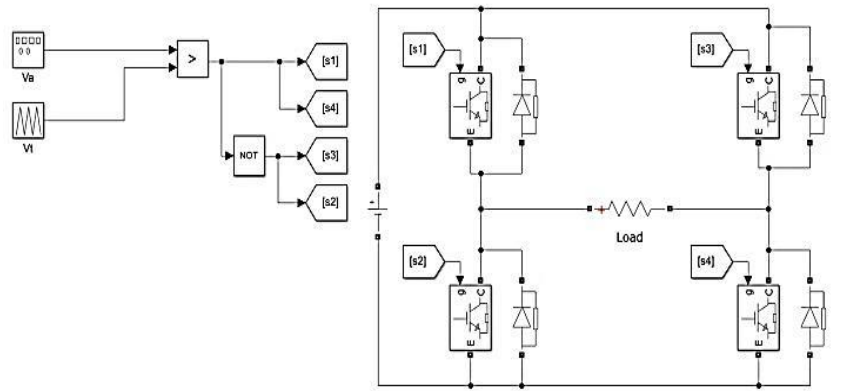

Şekil 3.1. Bipolar PWM Matlab/Simulink modeli

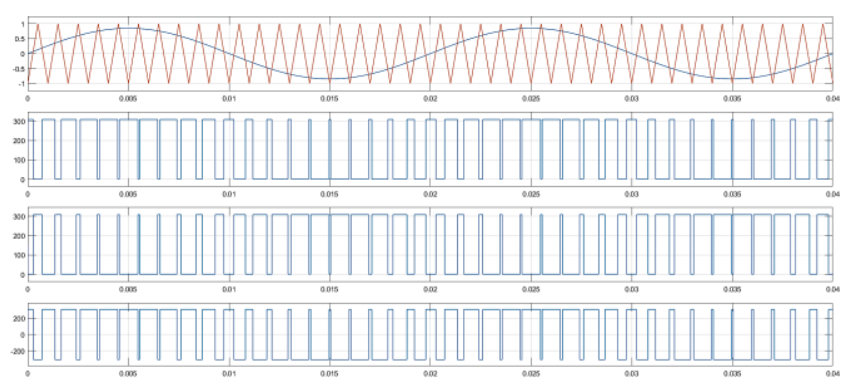

Şekil 3.2. Bipolar PWM dalga şekilleri

Şekil 3.3 ve Şekil 3.4'te sırasıyla unipolar PWM kontrol tekniği için oluşturulan Matlab/Simulink modeli ve dalga şekilleri görülmektedir.
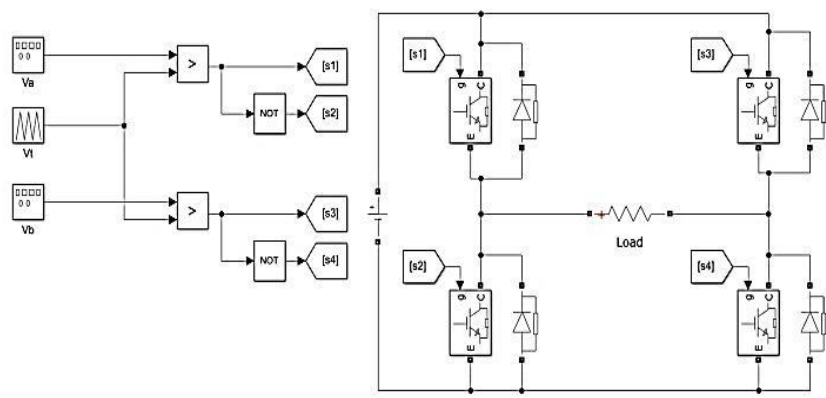

Şekil 3.3. Unipolar PWM Matlab/Simulink modeli

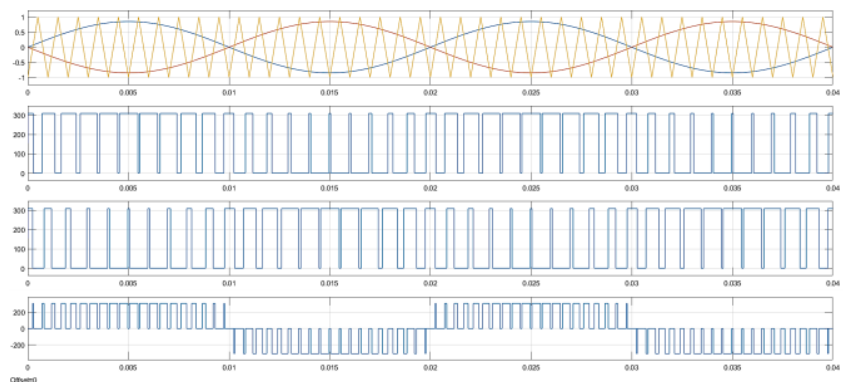

Şekil 3.4. Unipolar PWM dalga şekilleri

Matlab/Simulink ortamında bipolar ve unipolar PWM kontrol tekniklerinde modülasyon indeks değerleri sırasıyla 0.85 ve 1 için FFT analizi yapılarak çıkış geriliminin toplam harmonik distorsiyonu (THD) karşılaştırmalı olarak incelenmiştir. Bipolar PWM tekniğinde farklı modülasyon indekslerinde THD değerleri Şekil 3.5 ve Şekil 3.6'da verildiği üzere sırasıyla \%133.61 ve \%100.43 olmaktadır. Aynı şartlar altında unipolar PWM tekniğinde THD değerleri Şekil 3.7 ve Şekil 3.8'de görüldüğü üzere sirasıyla \%71.41 ve \%52.32 olarak bulunmuştur.

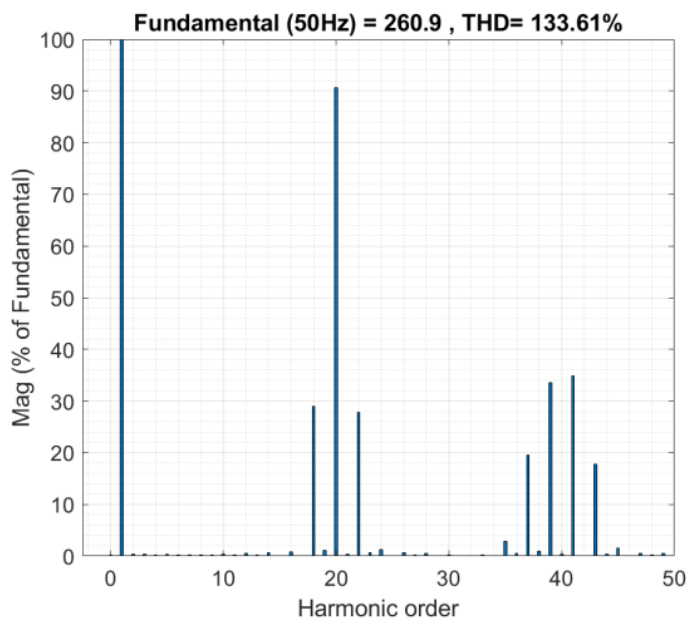

Şekil 3.5. Bipolar PWM FFT analizi (M=0.85)

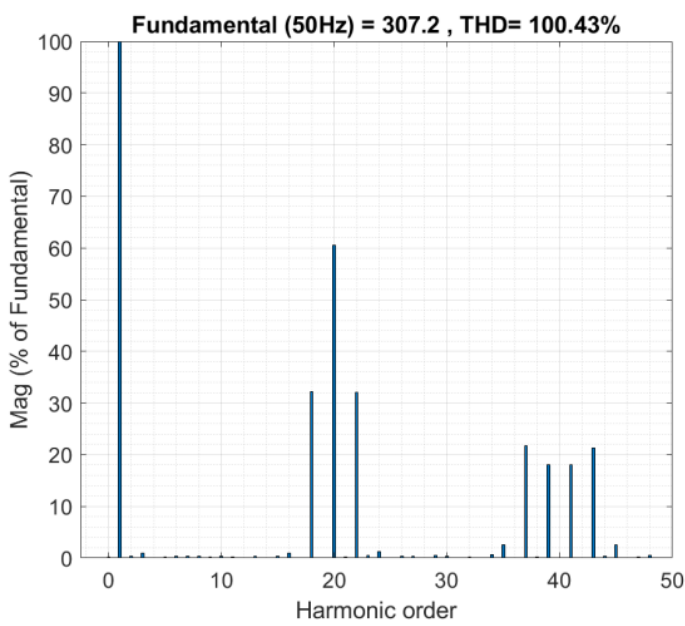

Şekil 3.6. Bipolar PWM FFT analizi $(M=1)$ 


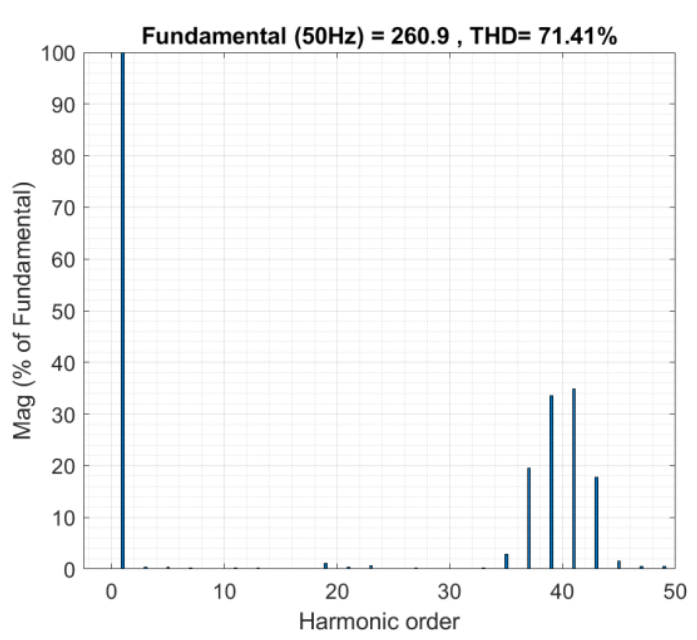

Şekil 3.7. Unipolar PWM FFT analizi $(\mathrm{M}=0.85)$

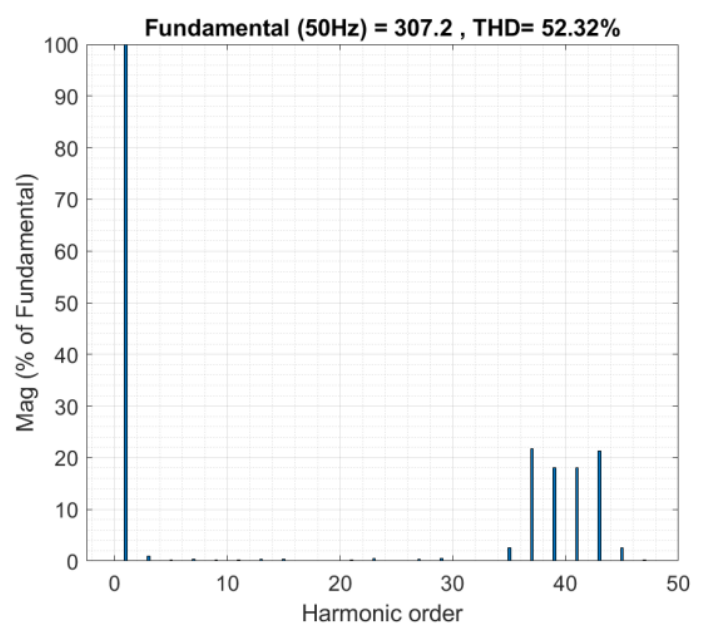

Şekil 3.8. Unipolar PWM FFT analizi $(\mathrm{M}=1)$

Benzetim çalışmalarında elde edilen veriler neticesinde çıkış gerilim sinyalinin kalitesi bakımından unipolar PWM tekniğinin bipolar PWM tekniğine göre her iki modülasyon indeks değerinde daha etkili olduğu görülmüştür. Unipolar PWM tekniği kullanılarak elde edilen gerilim sinyalinin THD değerlerinin daha düşük olması, unipolar PWM çıkışındaki anahtarlama frekansının bipolar PWM çıkışının iki katı olmasına dayanmaktadır. Bunun temel nedeni unipolar PWM tekniğinde tetikleme sinyallerini üretmek amaciyla iki adet düşük frekanslı sinüzoidal referans sinyali kullanılmasındandır. Anahtarlama frekansı arttıkça gerilim sinyalinin THD değeri düşmektedir. Her iki kontrol tekniğinde de modülasyon indeksinin artmasına bağlı olarak çıkış geriliminin THD değeri azalmıştır. Uygulanan modülasyon indeks değerinin büyük seçilmesi hem çıkış sinyal kalitesini iyileştirmekte hem de DC bara kullanım oranını arttırmaktadır.

Unipolar PWM tekniğinin bir diğer avantajı dv/dt oranının bipolar PWM tekniğine göre daha düşük olmasıdır. Şekil 3.2 ve Şekil 3.4 incelendiğinde çıkış gerilim dalga şekli bipolar PWM tekniğinde tüm periyot boyunca $+310 \mathrm{~V}$ ile $-310 \mathrm{~V}$ arasında değişirken, unipolar PWM tekniğinde ilk yarı periyotta $+310 \mathrm{~V}$ ile $0 \mathrm{~V}$ ve ikinci yarı periyotta $0 \mathrm{~V}$ ile $-310 \mathrm{~V}$ arasinda değişmektedir. $\mathrm{Bu}$ nedenle inverter katında kullanılan yarı iletken anahtarlar üzerindeki gerilim stresi unipolar PWM tekniği kullanılarak önemli ölçüde azaltılmaktadır.

\section{Sonuç}

$\mathrm{Bu}$ çalışmada bipolar ve unipolar PWM kontrol teknikleri aynı şartlar altında benzetim çalışmaları yapılarak karşılaştırmalı olarak incelenmiştir. Matlab/Simulink ortamında ilgili bloklar kullanılarak modelleri oluşturulan bipolar ve unipolar PWM teknikleri farklı modülasyon indeks değerlerinde tek fazlı tam köprü inverter devresine uygulanmıştır. Tam köprü inverter devresinde çıkış gerilim kalitesi incelendiğinde unipolar PWM tekniğinin her iki modülasyon indeksinde düşük THD değeri ile daha etkili olduğu görülmüştür. THD değerinin düşük olması harmoniklerin azaltılması için ihtiyaç duyulan filtre elemanlarının boyutlarının küçülmesine imkân verecektir.

Unipolar PWM tekniğinde $\mathrm{dv} / \mathrm{dt}$ oranının azalması ise inverter katındaki gerilim stresinin azalmasını sağlayacağından daha düşük nominal değerli yarı iletken anahtarların seçilmesi mümkün olacaktır. Sonuç olarak unipolar PWM tekniği kullanılan DC-AC dönüştücü devrelerinde hem çıkış gerilim kalitesi THD değerinin düşmesine bağlı olarak arttırılmakta hem de düşük nominal değerli anahtarların kullanımına imkan vermesinden dolayı oluşturulan devrenin maliyeti azaltılmaktadır.

\section{Kaynakça}

Endiz, M. S., Erel, S., \& Yagci, M. (2015). Comparative study of single phase power inverters based on efficiency and harmonic analysis. i-Manager's Journal on Instrumentation \& Control Engineering, 4(1), 1.

Endiz, M. S., \& Akkkaya, R. (2020, June). A Novel Single Phase Modified Quasi-Z-Source Inverter Circuit Design and Analysis. In 2020 24th International Conference Electronics (pp. 1-6). IEEE.

Engin, M., \& Gülersoy, T. (2018). Hibrid güç sistemleri için evirici tasarımı. Avrupa Bilim ve Teknoloji Dergisi, (14), 228-234.

Giri, S. K., Chakrabarti, S., Banerjee, S., \& Chakraborty, C. (2016). A carrier-based PWM scheme for neutral point voltage balancing in three-level inverter extending to full power factor range. IEEE Transactions on Industrial Electronics, 64(3), 1873-1883.

González, R., Lopez, J., Sanchis, P., \& Marroyo, L. (2007). Transformerless inverter for single-phase photovoltaic systems. IEEE Transactions on Power Electronics, 22(2), 693-697.

Kabalc1, E. (2020). Review on novel single-phase gridconnected solar inverters: Circuits and control methods. Solar Energy, 198, 247-274.

Khluabwannarat, P., Thammarat, C., Tadsuan, S., \& Bunjongjit, S. (2007, December). An analysis of iron loss supplied by sinusoidal, square wave, bipolar PWM inverter and unipolar PWM inverter. In 2007 International Power Engineering Conference (IPEC 2007) (pp. 1185-1190). IEEE.

Llorente, S., Monterde, F., Burdio, J. M., \& Acero, J. (2002, March). A comparative study of resonant inverter topologies used in induction cookers. In APEC. Seventeenth Annual IEEE Applied Power Electronics Conference and Exposition (Cat. No. 02CH37335) (Vol. 2, pp. 1168-1174). IEEE. 
Namboodiri, A., \& Wani, H. S. (2014). Unipolar and bipolar PWM inverter. International Journal for Innovative Research in Science \& Technology, 1(7), 237-243.

Sharma, C. S., Patel, N., \& Chaturvedi, H. (2016). Unipolar and Bipolar PWM Inverter Fed Induction Motor. no, 12, 16611664.

Soomro, J., Memon, T. D., \& Shah, M. A. (2016, November). Design and analysis of single phase voltage source inverter using Unipolar and Bipolar pulse width modulation techniques. In 2016 International Conference on Advances in Electrical, Electronic and Systems Engineering (ICAEES) (pp. 277-282). IEEE.

Wu, T. F., Kuo, C. L., Sun, K. H., \& Hsieh, H. C. (2013). Combined unipolar and bipolar PWM for current distortion improvement during power compensation. IEEE Transactions on Power Electronics, 29(4), 1702-1709.

Zope, P. H., Bhangale, P. G., Sonare, P., \& Suralkar, S. R. (2012). Design and Implementation of carrier based Sinusoidal PWM Inverter. International Journal of advanced research in electrical, electronics and instrumentation engineering, 1(4), 230-236. 